\title{
Factors underlying the changing of antibiotics in a paediatric intensive care unit in Pietermaritzburg, KwaZulu-Natal
}

\author{
A Vather, ${ }^{1,2}$ MB ChB, DCH, FCPaed; N H McKerrow, ${ }^{1,3}$ FCPaed (SA), MMed (Paed); M E Morgan, ${ }^{1,2}$ FCPaed (SA), Cert Crit Care (Paed) \\ ${ }^{1}$ Department of Paediatrics and Child Health, Nelson R Mandela School of Medicine, University of KwaZulu-Natal, Durban, South Africa \\ ${ }^{2}$ Department of Paediatrics, Pietermaritzburg Metropolitan Hospitals Complex, KwaZulu-Natal Department of Health, South Africa \\ ${ }^{3}$ Department of Health, KwaZulu-Natal, South Africa
}

Corresponding author: A Vather(ashaylan_v@yahoo.com)

\begin{abstract}
Background. Antibiotics are among the most commonly used drugs in a paediatric intensive care unit (PICU). Despite guidelines and protocols for the use of antibiotics, inappropriate use may contribute to an increase in antibiotic resistance. The factors behind changes in antibiotic prescriptions in the PICU at Grey's Hospital are unknown.

Objective. To establish the frequency, process and rationale behind antibiotic prescription changes in the PICU.

Methods. A retrospective descriptive study of all eligible patients admitted to the PICU during a 6-month period.

Results. Three-quarters of patients admitted to the PICU received antibiotics during their stay. The antibiotic prescription was changed in $80(58 \%)$ of the138 patients, with most changes (63.4\%) occurring within 3 days of admission. Patients younger than 1 year and those who were malnourished accounted for $57 \%$ of the changes. The majority (65\%) of the changes entailed the escalation of antibiotics and $89 \%$ of these were empiric therapy. De-escalation accounted for $35 \%$ of the changes. The rationale for a prescription change was not documented in $80 \%$ of cases.

Conclusion. Antibiotic use in this PICU and changes to prescriptions were common. Changes were generally made on an empirical basis soon after admission and were more likely to occur in young malnourished children and patients admitted for a medical reason or surgical emergency.

S Afr J Child Health 2018;12(3):143-147. DOI:10.7196/SAJCH.2018.v12i3.1466
\end{abstract}

Antibiotics have a critical role in the management of bacterial infections and reducing morbidity and mortality in critically-ill patients. ${ }^{[1]}$ They are therefore among the most frequently prescribed drugs in an intensive care unit (ICU), with a prescription rate as high as $73.4 \% .^{[2]}$

Principles that guide the appropriate use of antibiotics include making an accurate diagnosis, choosing the correct antibiotic, initiating therapy in time, prescribing the correct dose and de-escalating the treatment appropriately to achieve the narrowest spectrum and shortest duration of therapy. ${ }^{[3]}$ According to guidelines from the Council for Appropriate and Rational Antibiotic Therapy (CARAT) and the Surviving Sepsis Campaign, antibiotic treatment should be commenced after appropriate investigation and in all cases the patient must be followed up to ensure that a good clinical response is achieved and that microbial sensitivity to the antibiotic is confirmed. ${ }^{[4,5]}$

Despite these guidelines, $31-77 \%$ of antibiotic prescriptions in ICUs are reported to be inappropriate. ${ }^{[6-8]}$ This misuse of antibiotics contributes to higher rates of antimicrobial resistance and, in turn, increased morbidity, mortality and hospitalisation costs. ${ }^{[9,10]}$ Factors contributing to the development of antimicrobial resistance include: prolonged duration of antibiotic administration; the use of multiple, inappropriate or unnecessary antibiotics; suboptimal dosing; and failure to de-escalate treatment. ${ }^{[7]}$ The decision to change antibiotics should be directed by clinical findings or the results of investigations. ${ }^{[11-13]}$

Although antibiotic use in ICUs is well described, both locally and abroad, ${ }^{[1,2,6,9]}$ the rationale for changing antibiotics in a paediatric intensive care unit (PICU) is not. The present study considered the frequency, nature, timing and rationale behind changing antibiotic prescriptions after admission to a PICU at a tertiary hospital in KwaZulu-Natal, South Africa (SA).

\section{Methods}

Grey's Hospital is a tertiary institution in Pietermaritzburg, SA, which serves 3.5 million people. The PICU caters for medical and surgical patients under the care of the paediatric department. Medical officers and consultants provide 24-hour cover, with the latter being off-site after hours (between 16:30 and 08:00 on weekdays and all day on public holidays and weekends).

A retrospective chart review was conducted based on the records of all patients admitted to the PICU at Grey's Hospital between 1 January and 30 June 2013. Patients whose antibiotic prescriptions were changed during their stay in the PICU were included in the study.

During the study period, the PICU was an eight-bed unit, with capacity to ventilate up to four patients simultaneously. Weekend rounds were performed by the on-call team rather than the team assigned to the ICU. A paediatric intensivist was involved in the management of the unit during the study period, but there was no paediatric infectious diseases specialist. Established local protocols were followed for antibiotic use.

Every antibiotic changed while the patient was in the care of the PICU, or on the recommendation of the PICU staff during the period between acceptance and admission, was considered an event. Events included the addition of another antibiotic to the current regimen, subtraction of one or more antibiotics while continuing with others as prescribed, replacement of one or more antibiotics, and discontinuation of all antibiotics. Adding an antibiotic to the treatment regimen or replacing one with another that had a broader spectrum of cover was considered an escalation. De-escalation involved the subtraction of an antibiotic or replacement with one of a narrower spectrum, or complete discontinuation of antibiotic treatment. More than one event could be recorded for a patient during an admission. 
In cases for which no rationale for an event was recorded, reasons were inferred according to one of the following categories:

- clinical - temperature instability or deterioration or lack of improvement in the patient's condition

- laboratory - culture results or worsening septic markers (C-reactive protein, white cell count, platelet count, procalcitonin)

- imaging - chest X-rays, computed tomography scans or suggestive abdominal ultrasound images

- other - difficult intravenous access, complication or contraindication for an antibiotic or non-availability of intravenous antibiotic.

Details of the demographic, health and disease profile of the patient at and during admission, as well as details of antibiotic prescriptions, were extracted from the records. HIV status at admission was documented, with an infected status noted in the case of a positive polymerase chain reaction test in a child younger than 18 months or a positive ELISA result in a child older than 18 months. HIV status was noted as unknown if no documentation was included in the patient record or if the patient was described as an HIV-exposed child but no confirmed result was noted.

The data were collated and entered into a spreadsheet on a secured personal computer. All data were anonymised to ensure patient confidentiality; folder numbers were used to ensure no duplicate records were included in the study. Patients were grouped according to the number of events (none; single; multiple).

All statistical analyses were performed using SPSS (version 24). These included descriptive statistics, presented as frequency and comparative tables. A chi-squared test and Fisher's exact test were used to analyse categorical variables. A significance level of $p<0.05$ was used. A $95 \%$ confidence interval was used.

The Biomedical Research Ethics Committee, University of KwaZulu-Natal, granted ethical approval for the study (ref. no. BE188/13).

\section{Results}

There were 230 admissions to the PICU during the study period (Fig. 1). Of these, 184 patients had complete records, 3 had incomplete records and 43 records were missing. A total of 131 events were recorded from 80 patients with complete records.

During their stay in the ICU, 138 patients $(75 \%)$ received antibiotics. Of the 122 patients who were already on antibiotics at admission, 64 (52.4\%) had their prescription changed at least once. Of the 62 patients not on antibiotics at admission, 16 (25.8\%) subsequently required antibiotics.

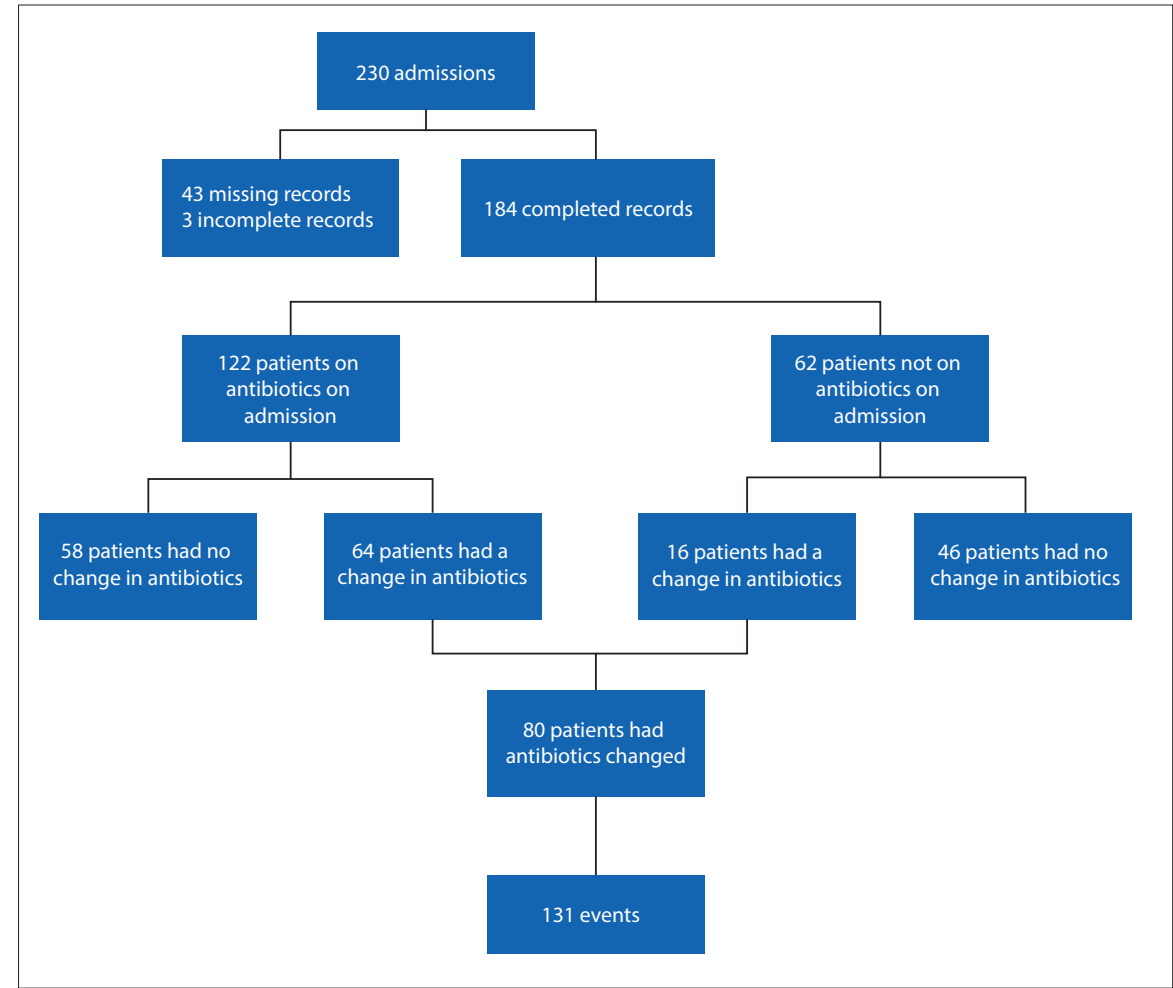

Fig. 1. Study population flow chart.

\section{Demographic profile of the study population}

Baseline characteristics of the 184 patients with complete records are presented in Table 1. The median age across the total group was 48.6 months. The median age of patients for whom events were recorded was significantly lower (14.5 months) than of those without events (48.0 months) $(p<0.001)$. The majority of patients were male (55.4\%). Approximately equal numbers of medical and surgical cases were admitted. Surgical admissions included 46 elective cases and 44 emergency cases, of which 10 were trauma related.

The nutritional status of patients younger than 5 years in this group was assessed using weight-for-age $Z$-scores. Of them, 79 (63.2\%) had a normal nutritional status, 35 (28\%) were underweight and $1(0.8 \%)$ was overweight. Body weight was not recorded in $10(8 \%)$ cases. On admission, 84 (45.7\%) patients were HIV negative, with the rest either being HIV positive $(n=27 ; 14.7 \%)$ or having an unknown HIV status $(n=73 ; 39.6 \%)$.

Antibiotic changes were more likely to be seen in younger $(p<0.001)$ or underweight patients $(p=0.237)$, although the latter result was not statistically significant. Gender and HIV status were not significant determinants of an antibiotic change. Surgical patients were generally less likely to have had an event $(p=0.035)$, likely driven by elective rather than emergency cases $(p<0.001)$. This is possibly a result of the average length of stay (1.9 days for elective surgical cases compared with 4.6 days for both emergency surgical and medical patients $(p \leq 0.001))$.

\section{Details of antibiotic changes}

Single changes accounted for the majority of events $(n=50 ; 62.5 \%)$. Replacements (28.2\%) and additions (41.9\%) were the most common.

As shown in Table 2A, most events occurred during routine working hours $(72.5 \%)$ and within the first 3 days of admission (63.4\%). Of the 55 replacements, 48 involved changing from narrow- to broadspectrum antibiotics. Escalation (additions and replacements of narrow to broad spectrum combined) therefore accounted for $65 \%$ of events. After-hour events were more likely to be escalations than de-escalations.

Only 27 events (20.6\%) had a documented rationale. Notably, no rationale was documented for $104(79.4 \%)$ of the events $(p<0.001)$. For these, the clinical records associated with the event were reviewed to infer the reasons for the change, which led to 149 inferred reasons (Table 2B); some events were associated with more than one reason. Antibiotics were more likely to be revised in response to clinical findings $(n=65 ; 36.9 \%)$ or laboratory results $(n=36 ; 20.5 \%)$, while the completion of antibiotics accounted for most of the de-escalations.

\section{Demographic profile across event groups}

Table 3 shows the demographic profile of patients who received a single antibiotic 
Table 1. Baseline characteristics of study population

\begin{tabular}{|c|c|c|c|c|}
\hline Characteristics & All $(N=184), n(\%)$ & $\begin{array}{l}\text { Antibiotic changed }(N=80), \\
n(\%)\end{array}$ & $\begin{array}{l}\text { No change in antibiotic }(N=104), \\
n(\%)\end{array}$ & $p$-value \\
\hline Age (months), median (IQR) & $48.6(0.5-168)$ & $14.5(0.5-156)$ & $48.0(1-168)$ & $<0.001$ \\
\hline \multicolumn{5}{|l|}{ Gender } \\
\hline Male & $102(55.4)$ & $45(56.2)$ & $57(54.8)$ & 0.235 \\
\hline Female & $82(44.6)$ & $35(43.8)$ & $47(45.2)$ & 0.185 \\
\hline \multicolumn{5}{|l|}{ Admitting discipline } \\
\hline Medical & $94(51.1)$ & $45(56.2)$ & $49(47.1)$ & 0.680 \\
\hline Surgical & $90(48.9)$ & $35(43.8)$ & $55(52.9)$ & 0.035 \\
\hline Elective & $46(51.1)$ & $11(31.4)$ & $35(63.6)$ & $<0.001$ \\
\hline Emergency & $44(48.9)$ & $24(68.6)$ & $20(36.4)$ & 0.546 \\
\hline \multicolumn{5}{|c|}{$\begin{array}{l}\text { Relative weight for patients }<60 \text { months } \\
(N=125)\end{array}$} \\
\hline Underweight & $35(28.0)$ & $21(33.3)$ & $14(22.6)$ & 0.237 \\
\hline Normal & $79(63.2)$ & $35(55.6)$ & $44(70.9)$ & 0.311 \\
\hline Overweight & $1(0.8)$ & $1(1.6)$ & $\mathrm{n} / \mathrm{a}$ & 0.180 \\
\hline Unknown & $10(8.0)$ & $6(9.5)$ & $4(6.5)$ & 0.247 \\
\hline \multicolumn{5}{|l|}{ HIV status } \\
\hline Positive & $27(14.7)$ & $15(18.8)$ & $12(11.5)$ & 0.564 \\
\hline Negative & $84(45.7)$ & $37(46.2)$ & $47(45.2)$ & 0.275 \\
\hline Unknown & $73(39.6)$ & $28(35.0)$ & $45(43.3)$ & 0.047 \\
\hline
\end{tabular}

Table 2A. Timing of antibiotic changes

\begin{tabular}{|c|c|c|c|c|c|c|}
\hline Parameter & $\begin{array}{l}\text { All }(N=131), \\
n(\%)\end{array}$ & $\begin{array}{l}\text { Addition } \\
(N=37), n(\%)\end{array}$ & $\begin{array}{l}\text { Subtraction } \\
(N=14), n(\%)\end{array}$ & $\begin{array}{l}\text { Replacement } \\
(N=55), n(\%)\end{array}$ & $\begin{array}{l}\text { Discontinuation } \\
(N=25), n(\%)\end{array}$ & $p$-value \\
\hline \multicolumn{7}{|l|}{ Time of change } \\
\hline Working hours & $95(72.5)$ & $23(62.2)$ & $13(92.9)$ & $35(63.6)$ & $24(96.0)$ & 0.001 \\
\hline After hours & $36(27.5)$ & $14(37.8)$ & $1(7.1)$ & $20(36.4)$ & $1(4.0)$ & \\
\hline \multicolumn{7}{|c|}{ Time since admission } \\
\hline$<24$ hours & $41(31.3)$ & $14(37.8)$ & $3(21.4)$ & $22(40.0)$ & $2(8.0)$ & 0.001 \\
\hline 1 - 3 days & $42(32.1)$ & $18(48.7)$ & $3(21.4)$ & $19(34.5)$ & $2(8.0)$ & \\
\hline 4 - 7 days & $33(25.2)$ & $2(5.4)$ & $8(57.2)$ & $9(18.2)$ & $14(56.0)$ & \\
\hline$>7$ days & $15(11.4)$ & $3(8.1)$ & $0(0.0)$ & $5(7.3)$ & $7(28.0)$ & \\
\hline
\end{tabular}

Table 2B. Rationales for antibiotic changes

\begin{tabular}{|c|c|c|c|c|c|c|}
\hline Rationale & $\begin{array}{l}\text { All }(N=176), \\
n(\%)\end{array}$ & $\begin{array}{l}\text { Addition } \\
(N=54), n(\%)\end{array}$ & $\begin{array}{l}\text { Subtraction } \\
(N=14), n(\%)\end{array}$ & $\begin{array}{l}\text { Replacement } \\
(N=82), n(\%)\end{array}$ & $\begin{array}{l}\text { Discontinuation } \\
(N=26), n(\%)\end{array}$ & $p$-value \\
\hline Clinical & $65(36.9)$ & $25(46.3)$ & $0(0.0)$ & $40(48.8)$ & $0(0.0)$ & \\
\hline Laboratory & $36(20.5)$ & $16(29.6)$ & $0(0.0)$ & $20(24.3)$ & $0(0.0)$ & $<0.0001$ \\
\hline Imaging & $11(6.3)$ & $3(5.6)$ & $0(0.0)$ & $8(9.8)$ & $0(0.0)$ & \\
\hline Surgical & $15(8.5)$ & $9(16.7)$ & $0(0.0)$ & $6(7.3)$ & $0(0.0)$ & \\
\hline Other & $18(10.2)$ & $1(1.8)$ & $5(35.7)$ & $8(9.8)$ & $4(16.4)$ & \\
\hline Completion & $31(17.6)$ & $0(0.0)$ & $9(64.3)$ & $0(0.0)$ & $22(84.6)$ & \\
\hline
\end{tabular}

change compared with those who received multiple changes. Most events were recorded in infants younger than 1 year $(n=46 ; 57.4 \%)$. In addition, infants comprised the majority of cases for whom multiple events were recorded (76.7\%). Multiple events were also more common in medical and underweight patients; however, only age was a statistically significant factor $(p=0.025)$.

\section{Discussion}

Antibiotics are integral to the management of critically ill patients admitted to an ICU. Given the high prescription rate ${ }^{[1]}$ and risk of antimicrobial resistance, it is important to understand the timing and rationale behind antibiotic prescription practices.
Antibiotics were administered to $75 \%$ of our study population during their stay in the PICU. This is less than what is reported from India $(100 \%)^{[14]}$ and China $(95 \%){ }^{[15]}$ but comparable to the Canadian experience $(70-79 \%) .{ }^{[16]}$ This could be attributed to the nature of our PICU, which includes both high-care and ICU beds. Many patients may therefore be admitted for observation, but would not require antibiotics.

Age was identified as a significant predictor of antibiotic change in this population. Although the median age of patients for whom events were recorded was 48.6 months ( 4.5 years), the majority (57.4\%) of these patients were younger than 1 year. Bhullar et al. ${ }^{[17]}$ reported a similar finding $(42.8 \%)$. As $85 \%$ of the underweight 
Table 3. Comparison of demographic characteristics of patients receiving single or multiple antibiotic changes

\begin{tabular}{|c|c|c|c|c|}
\hline Characteristics & $\begin{array}{l}\text { Total } \\
(N=80), n(\%)\end{array}$ & $\begin{array}{l}\text { Single event } \\
(N=50), n(\%)\end{array}$ & $\begin{array}{l}\text { Multiple event } \\
(N=30), n(\%)\end{array}$ & $p$-value \\
\hline \multicolumn{5}{|l|}{ Age (years) } \\
\hline$<1$ & $46(57.4)$ & $23(46.0)$ & $23(76.7)$ & \multirow[t]{3}{*}{0.025} \\
\hline $1-5$ & $17(21.3)$ & $13(26.0)$ & $4(13.3)$ & \\
\hline$>5$ & $17(21.3)$ & $14(28.0)$ & $3(10.0)$ & \\
\hline \multicolumn{5}{|l|}{ Gender } \\
\hline Male & $45(56.3)$ & $27(54.0)$ & $18(60.0)$ & \multirow[t]{2}{*}{0.647} \\
\hline Female & $35(43.7)$ & $23(46.0)$ & $12(40.0)$ & \\
\hline \multicolumn{5}{|l|}{ HIV status } \\
\hline Positive & 15 (18.7) & $9(18.0)$ & $6(20.0)$ & \multirow[t]{3}{*}{0.910} \\
\hline Negative & $37(46.3)$ & $24(48.0)$ & $13(43.3)$ & \\
\hline Unknown & $28(35.0)$ & $17(34.0)$ & $11(36.7)$ & \\
\hline \multicolumn{5}{|l|}{ Weight } \\
\hline Underweight & $21(33.3)$ & $9(25.0)$ & $12(44.4)$ & \multirow[t]{4}{*}{0.332} \\
\hline Normal & $35(55.6)$ & $23(63.9)$ & $12(44.4)$ & \\
\hline Overweight & $1(1.6)$ & $0(0.0)$ & $1(3.7)$ & \\
\hline Unknown & $6(9.5)$ & $4(11.1)$ & $2(7.5)$ & \\
\hline \multicolumn{5}{|c|}{ Admitting discipline } \\
\hline Medical & $45(56.3)$ & $24(48.0)$ & $21(70.0)$ & \multirow[t]{2}{*}{0.065} \\
\hline Surgical & 35 (43.7) & $26(52.0)$ & $9(30.0)$ & \\
\hline
\end{tabular}

patients for whom events were recorded were infants $(n=18 / 21)$ and infants were more likely to have multiple events, it is possible that these were two confounding factors. In a study conducted in the same unit as ours, Hendricks et al ${ }^{[18]}$ noted that the highest rate of malnutrition and death was reported for children younger than 1 year, suggesting an 'increased vulnerability in this age group and possibly the need to practise early aggressive intervention in infants admitted to an ICU to improve outcomes'. It is possible that, in the cases reviewed during our study, infants were also considered to be more vulnerable by the attending doctor, who therefore applied a lower threshold for reviewing prescriptions.

In SA, the HIV epidemic has led to a rise in the incidence of bacterial infections and subsequently increased admissions to the PICU of children with pneumonia or gastroenteritis. ${ }^{[19]}$ While HIV-infected patients were more likely to have had their antibiotic prescription changed, our analysis did not show HIV status to be a significant factor. This finding is possibly due to the large number of patients with an unknown HIV status and the small number in the HIV-positive group.

Medical patients were more likely to receive antibiotics and experience multiple events than surgical patients. On further breakdown, elective surgical cases were less likely than emergency cases to be prescribed antibiotics or experience a change in their prescription. This could be because elective surgical patients were less sick on admission than emergency cases or because of a shorter stay in the ICU.

Antibiotic prescriptions were changed in more than half (58\%) of the patients who were already on antibiotics at admission to the unit. Two-thirds (65\%) of these changes entailed an escalation rather than a de-escalation of antibiotics. Most changes (89\%) occurred on an empirical basis in response to the clinical condition of the patient, supported by laboratory, radiological or surgical findings. This incidence is higher than what has been reported in India $(42 \%),{ }^{[14]}$ China $(72 \%)^{[15]}$ or Canada $(27-40 \%) \cdot{ }^{[16]}$ However, the inferred reasons for changing antibiotics were in keeping with international guidelines. ${ }^{[5,11-13]}$ Directed therapy accounted for $5.5 \%$ of antibiotic prescription changes, which is lower than both the Indian $(15 \%)^{[14]}$ and Canadian $(34 \%)^{[16]}$ reports. The de-escalation of antibiotics accounted for $35 \%$ of the changes, which is in keeping with findings from other studies. ${ }^{[15,16]}$
A possible reason for the findings in this study is the absence of an antimicrobial stewardship programme in the PICU. Such programmes are usually coordinated by a team comprising an infectious disease specialist and a pharmacist, but can also include a microbiologist or clinician with an interest in antibiotic stewardship. Elements of these programmes include prospective audits and feedback on antimicrobial prescriptions to clinicians, formulary restriction, education (passive activities, conference presentations, student and in-house teaching efforts), use of clinical pathways and guidelines(antimicrobial cycling, antimicrobial order forms and combination therapy), de-escalation of therapy and intravenous to oral antimicrobial conversion when appropriate. ${ }^{[20]}$

Documentation of the reasons for changing antibiotic prescriptions was poor, with $79.4 \%$ of events not associated with a documented rationale $(p<0.001)$. Documentation regarding the duration of antibiotic use and culture results was also poor. Documenting the reason for an antimicrobial prescription is recommended for good clinical practice, ${ }^{[21]}$ as it ensures communication of the diagnosis between clinical teams and informs the appropriate review of treatment.

Most prescription changes (63.4\%) occurred within the first 3 days of admission to the PICU, with just less than half (49.4\%) occurring within the first 24 hours. Most of these were the escalation of antibiotics (65\%) and were most likely in response to the severity of the patient's clinical condition as assessed by the paediatrician.

The limitations of this study are that it was a retrospective, singlecentre study. Despite comparisons made with other studies, this study did not explore the appropriateness of the antibiotic changes, although the frequency and rationale for antibiotic changes were identified in keeping with the main aim of the study.

\section{Conclusion}

This study described factors associated with the change of antibiotic prescriptions following admission to a PICU. A revision of prescription was seen in a third of the patients, usually soon after admission and during working hours. The majority involved the escalation rather than de-escalation of antibiotics. These revisions were more likely to be empirical and to occur in young malnourished children and those admitted for surgical emergencies or medical conditions. The rationale for changes was infrequently documented. 
It is recommended that antimicrobial stewardship programmes be developed and implemented to improve the use of antibiotics in the PICU. In addition, antibiotic prescriptions need to be correlated with a documented rationale and recommended duration. Improved practice for antimicrobial use could be accomplished through continued education of staff, together with the development and implementation of a dedicated checklist as part of the documentation.

\section{Acknowledgements. None.}

Author contributions. AV, NHMcK and MEM designed the study and developed the methodology. AV collected the data, performed the analysis and prepared the manuscript. NHMcK and MEM supervised the process and reviewed and edited the manuscript.

Funding. None.

Conflicts of interest. None.

1. Ibrahim EH, Sherman G, Ward S, Fraser VJ, Kollef MH. The influence of inadequate antimicrobial treatment of bloodstream infections on patient outcomes in the ICU setting. Chest 2000;118(1):146-155. https://doi. org/10.1378/chest.118.1.146

2. Paruk F, Richards G, Scribante J, Bhagwanjee S, Mer M, Perrie H. Antibiotic prescription practices and their relationship to outcome in South African intensive care units: Findings of the Prevalence of Infection in South African Intensive Care Units (PISA) study. S Afr Med J 2012;102(7):613-616. https:// doi.org/10.7196/samj.5833

3. Leekha S, Terrell CL, Edson RS. General principles of antimicrobial therapy. Mayo Clin Proc 2011;86(2):156-167. https://doi.org/10.4065/ mcp.2010.0639

4. Slama TG, Amin A, Brunton SA, et al. A clinician's guide to the appropriate and accurate use of antibiotics: The Council for Appropriate and Rational Antibiotic Therapy (CARAT) criteria. Am J Med 2005;118(Suppl 7A):1S-6S. https://doi. org/10.1016/j.amjmed.2005.05.007

5. Surviving Sepsis Campaign. Surviving Sepsis Campaign: International guidelines for management of severe sepsis and septic shock: 2016. http://www. survivingsepsis.org/guidelines/ (accessed 1 August 2017).

6. Erbay A, Bodur H, Akinci E, Colpan A. Evaluation of antibiotic use in intensive care units of a tertiary care hospital in Turkey. J Hosp Infect 2005;59(1):53-61 https://doi.org/10.1016/j.jhin.2004.07.026

7. Von Gunten V, Reymond JP, Boubaker K, et al. Antibiotic use: Is appropriateness expensive? J Hosp Infect 2009;71(2):108-111. https://doi.org/10.1016/j. jhin.2008.10.026
8. Shorr AF, Micek ST, Kollef MH. Inappropriate therapy for methicillin-resistant Staphylococcus aureus: Resource utilization and cost implications. Crit Care Med 2008;36(8):2335-2340. https://doi.org/10.1097/ccm.0b013e31818103ea

9. Tünger O, Dinç G, Ozbakkaloglu B, Atman UC, Algün U. Evaluation of rational antibiotic use. Int J Antimicrob Agents 2000;15(2):131-135. https://doi. org/10.1016/s0924-8579(00)00158-8

10. Gaynes R. The impact of antimicrobial use on the emergence of antimicrobialresistant bacteria in hospitals. Infect Dis Clin North Am 1997;11(4):757-765. https://doi.org/10.1016/s0891-5520(05)70388-3

11. MacDougall C, Polk RE. Antimicrobial stewardship programs in health care systems. Clin Microbiol Rev 2005;18(4):638-656. https://doi.org/10.1128/ cmr.18.4.638-656.2005

12. Lim CJ, Kong DCM, Stuart RL. Reducing inappropriate antibiotic prescribing in the residential care setting: Current perspectives. Clin Interv Aging 2014;9:165177. https://doi.org/10.2147/CIA.S46058

13. Dryden M, Saeed K, Townsend R, et al. Antibiotic stewardship and early discharge from hospital: Impact of a structured approach to antimicrobial management J Antimicrob Chemother 2012;67(9):2289-2296. https://doi.org/10.1093/jac/ dks193

14. Abbas Q, Ul Haq A, Kumar R, Ali SA, Hussain K, Shakoor S. Evaluation of antibiotic use in pediatric intensive care unit of a developing country. Indian J Crit Care Med 2016;20(5):291-294. https://doi.org/10.4103/0972-5229.182197

15. Ding H, Yang Y, Chen Y, Wang Y, Fan S, Shen X. Antimicrobial usage in paediatric intensive care units in China. Acta Paediatr 2008;97(1):100-104. https://doi. org/10.1111/j.1651-2227.2007.00580.x

16. Blinova E, Lau E, Bitnun A, et al. Point prevalence survey of antimicrobial utilization in the cardiac and pediatric critical care unit. Pediatr Crit Care Med 2013;14(6):280-288. https://doi.org/10.1097/pcc.0b013e31828a846d

17. Bhullar HS, Shaikh FAR, Deepak R, Poddutoor PK, Chirla D. Antimicrobia justification form for restricting antibiotic use in a pediatric intensive care unit. Indian Pediatr 2016;53:304-306. https://doi.org/10.1007/s13312-016-0841-0

18. Hendricks CL, McKerrow NH, Hendricks RJ. Factors present on admission associated with increased mortality in children admitted to a paediatric intensive care unit (PICU). S Afr J Child Health 2016;10(1):57-62. https://doi.org/10.7196/ sajch.2016.v10i1.1048

19. Morrow BM, Argent AC. Ventilator-associated pneumonia in a paediatric intensive care unit in a developing country with high HIV prevalence. J Paediatr Child Health 2009;45(3):104-111. https://doi.org/10.1111/j.14401754.2008.01437.x

20. Dellit TH, Owens RC, McGowan JE, et al. Infectious Diseases Society of America and the Society for Healthcare Epidemiology of America guidelines for developing an institutional program to enhance antimicrobial stewardship. Clin Infect Dis 2007;44(2):159-177. https://doi.org/10.1086/510393

21. Kuhn T, Basch P, Barr M, Yackel T, Medical Informatics Committee of the American College of Physicians. Clinical documentation in the 21st century: Executive summary of a policy position paper from the American College of Physicians. Ann Intern Med 2015;162(4):301-303. https://doi.org/10.7326/m14-2128

Accepted 23 April 2018 